\title{
A NEW GRAPHICAL APPROACH TO CLASSIFY SPATIAL POINT PATTERNS BASED ON HIERARCHICAL CLUSTER ANALYSIS
}

\author{
Tsutomu Takai, Yoshiyasu Tamura ${ }^{\dagger}$ and Hitoshi Motoyama ${ }^{\ddagger}$
}

\begin{abstract}
In this paper, a novel graphical method to classify spatial point patterns is proposed. This method is called the AG-curve. It is constructed using the results of hierarchical cluster analysis, and it has many variations corresponding to the various agglomeration methods used in such analysis. The characteristics of the AGsi-curve, which is a variant of the AG-curve, are clarified and several applications to real data are shown. The classification performance of the AGsi-curve is compared with that of other graphical methods, and the difference between the AGsi-curve and the other methods is shown graphically.
\end{abstract}

\section{Introduction}

This paper proposes a new graphical method to classify spatial point patterns (SPPs) into three categories as follows:

aggregated patterns: if an attractive force acts among points;

regular patterns: if a repulsive force acts among points;

random patterns: if neither an attractive force nor a repulsive force acts among points.

The focus of this paper is to classify an observed SPP into any of the above three categories, and we do not consider whether the SPP has complete spatial randomness (CSR; Diggle, 1979). In most previous studies (e.g., Zimmerman, 1993), the analysis of an observed SPP is often begun with a formal test of a null hypothesis of CSR. After the null hypothesis is rejected, then the set is analyzed to determine whether it is regular or aggregated.

Analyses of SPPs have been performed in various fields, including ecology (e.g., Perry et al., 2006), epidemiology (e.g., Gatrell et al., 1996), seismology (e.g., Ogata, 1999), economics (e.g., Duranton \& Overman, 2005) and geography (e.g., Okabe \& Yamada, 2001), and in each such field, this has provided useful insight. Recently, the analytical methods for SPPs are extended to spatio-temporal analysis (Diggle, 2013) and multivariate SPPs (Waagepetersen et al., 2016). There are two important graphical methods that are similar to our proposed method: the $G$-function (Diggle, 2003) and the $K$-function (Ripley, 1977). Both of these methods have been used widely and can be implemented in a simplistic form, and their results can be interpreted intuitively.

\footnotetext{
* School of Multidisciplinary Sciences Department of Statistical Science, The Graduate University for Advanced Studies, 10-3 Midori-cho, Tachikawa, Tokyo 190-8562, Japan E-mail: ttakai@ism.ac.jp

$\dagger$ Department of Statistical Modeling, The Institute of Statistical Mathematics, The Graduate University for Advanced Studies, 10-3 Midori-cho, Tachikawa, Tokyo 190-8562, Japan E-mail: tamura@ism.ac.jp

$\ddagger$ College of Economics, Aoyama Gakuin University, 4-4-25 Shibuya, Shibuya-ku, Tokyo 150-8366, Japan

E-mail: hitoshi@aoyamagakuin.jp

Key words: AG-curve; AGsi-curve; spatial point patterns; hierarchical cluster analysis
} 
The $G$-function is the cumulative distribution function (CDF) of the point-to-point nearest-neighbor distance $d_{i}$, the distance from the $i$ th point to the next nearest point in some region. The theoretical $G$-function under CSR is $G(r)=1-\exp \left(-\pi \lambda r^{2}\right)$, where $\lambda$ is the intensity; its estimator is the empirical CDF $\hat{G}(r)=n^{-1} \sum_{d_{i} \leq r} 1$, where $n$ is the number of points in the region. $\hat{G}(r)$ is used to compare an observed SPP to the characteristics of CSR, as follows:

$$
\begin{aligned}
& \hat{G}(r)>1-\exp \left(-\pi \lambda r^{2}\right) \text { for an aggregated pattern } \\
& \hat{G}(r)<1-\exp \left(-\pi \lambda r^{2}\right) \text { for a regular pattern. }
\end{aligned}
$$

Unfortunately, as is commonly known and described later, the $G$-function cannot sometimes discriminate different types of SPPs because the connection based on the point-topoint nearest-neighbor distance does not always link all of the points in the region.

The $K$-function is a measure of the second-order properties of an SPP. Ripley (1977) defined the function $K(r)$ as follows: $\lambda K(r)$ is the expected number of further points within $r$ of an arbitrary point of the process.

When CSR holds, the theoretical $K$-function is $K(r)=\pi r^{2}$. Ripley's edge-corrected estimator of the $K$-function (Ripley, 1976; Dixon, 2002) is

$$
\begin{aligned}
& \hat{K}(r)=\hat{\lambda}^{-1} \sum_{i} \sum_{j \neq i} w\left(l_{i}, l_{j}\right)^{-1} \frac{I\left(d_{i j}<r\right)}{N}, \text { where } \\
& N: \text { the observed number of points in the study region } \\
& \hat{\lambda}: N / A \text {, where } A \text { is the area of the study region } \\
& d_{i, j}: \text { the distance between the } i \text { th and } j \text { th points } \\
& w\left(l_{i}, l_{j}\right): \text { the weight function for the edge correction } \\
& I\left(d_{i j}<r\right): \text { the indicator function, which is } 1 \text { if } d_{i, j}<r .
\end{aligned}
$$

$\hat{K}(r)$ in the small $r$ region is used to compare an observed SPP to the characteristics of CSR as follows:

$$
\begin{aligned}
& \hat{K}(r)>\pi r^{2} \text { for an aggregated pattern } \\
& \hat{K}(r)<\pi r^{2} \text { for a regular pattern. }
\end{aligned}
$$

As is well known, the $K$-function has weaknesses as follows. In some cases, the deviation from the CSR in the $K$-function appears only in a small region, so sometimes the $K$-function in a region must be emphasized in some way. Moreover the $K$-function is impacted by the edge effect without the edge correction.

In this paper, the agglomerative curve (AG-curve) is newly proposed to classify SPPs graphically. The AG-curve utilizes the dendrogram of a hierarchical cluster analysis in order to make up for the weaknesses of the $G$-function and the $K$-function. In other words, the AG-curve can grasp not only the local information of the SPP but also the overall information. Previous to this paper, Pereira (2003) independently proposed two methods using a hierarchical cluster analysis in her investigation of forest spatial point patterns. One of them is a knee plot of the fusion distances which is essentially the same as the AG-curve. The other is the fusion distance function, $H(t)$, which is the empirical cumulative distribution function of the fusion distances. Pereira (2003) mainly focused on $H(t)$, but the knee plot of the fusion distances was used only for exploratory data analysis without theoretical insights or applications to classifications. This paper places importance on the AG-curve and presents the mathematical background of the AG-curve that enables classification of SPPs.

The remainder of this paper is organized as follows. Section 2 defines the AG-curve and shows the methodology for classification of SPPs. Furthermore, it shows the results of classification for real SPPs using the AGsi-curve, which is a variant of the AG-curve. 
A New Graphical Approach to Classify Spatial Point Patterns

Section 3 compares the characteristics of the AGsi-curve to those of the $G$-function and the $K$-function graphically. Section 4 discusses the relationship between the AGsi-curve and the minimum spanning tree (MST; Kruskal, 1956; Gower and Ross, 1969). Finally, Section 5 gives the conclusion.

\section{The AG-curve}

\subsection{Definition}

The proposed graphical method is based on the results of a hierarchical cluster analysis of SPPs, and we define the AG-curve by converting the agglomerative information contained in the dendrogram of the hierarchical cluster analysis and express it as

$$
h(k) \quad(k=1,2, \ldots, n-1),
$$

where $k$ is the number of clusters, and $n$ is the number of observations. Note that $h(k)$ is the largest ultrametric distance (Johnson, 1967) among $k$ clusters after agglomeration by the hierarchical cluster algorithm. In other words, $h(k)$ is the distance between two merged clusters at the $(n-k)$ th clustering stage to join the two nearest clusters. When $k=1$ at the final clustering stage, $h(k)$ takes the maximum value, and when $k=n-1$ at the first clustering stage, $h(k)$ takes the minimum value. Thus, the AG-curve is a monotonically decreasing curve.

The AG-curve depends on using a hierarchical cluster algorithm, such as single-linkage cluster analysis (SLCA), complete-linkage cluster analysis (CLCA), or average-linkage cluster analysis (ALCA) (Lance and Williams, 1966 \& 1967). Therefore, we can define different variants of the AG-curve depending on which hierarchical cluster algorithm is used. We refer to the AG-curve based on SLCA, CLCA, and ALCA as the AGsi-curve, AGco-curve, and AGav-curve, respectively.

The AG-curve is obtained as a result of plots using the following steps.

[Step 1] Compute the distance matrix for all objects being clustered.

[Step 2] Perform a hierarchical cluster analysis using the distance matrix computed in Step 1.

[Step 3] Evaluate the height $h(k)$ at which each merger was made in Step 2.

[Step 4] Obtain the number of clusters corresponding to the height evaluated in Step 3.

[Step 5] Plot the number of clusters along the horizontal axis and the height along the vertical axis.

A simple example of an AGsi-curve is shown in Figure 1. It was produced using the above procedure with the Euclidean distance. The upper-left panel shows an SPP that has ten points in a unit square; this panel also shows the results of an SLCA. The lower-left panel shows the AGsi-curve for the points in the upper-left panel. The right panel shows the dendrogram corresponding to the AGsi-curve.

\subsection{Application to classification of SPPs}

Our proposed method allows us to statistically analyze an SPP by using the distribution of the AG-curve under CSR. After determining that CSR does not hold for a particular SPP, the method can determine whether the SPP is more likely to be regular or aggregated. For simplicity, we use the simple Euclidean measure of the distance between points. In the remainder of this paper, we will focus almost exclusively on the AGsi-curve, because it is mathematically similar to the $G$-function and the mathematical relation between $h(k)$ and MST is known in case of the single linkage method. 

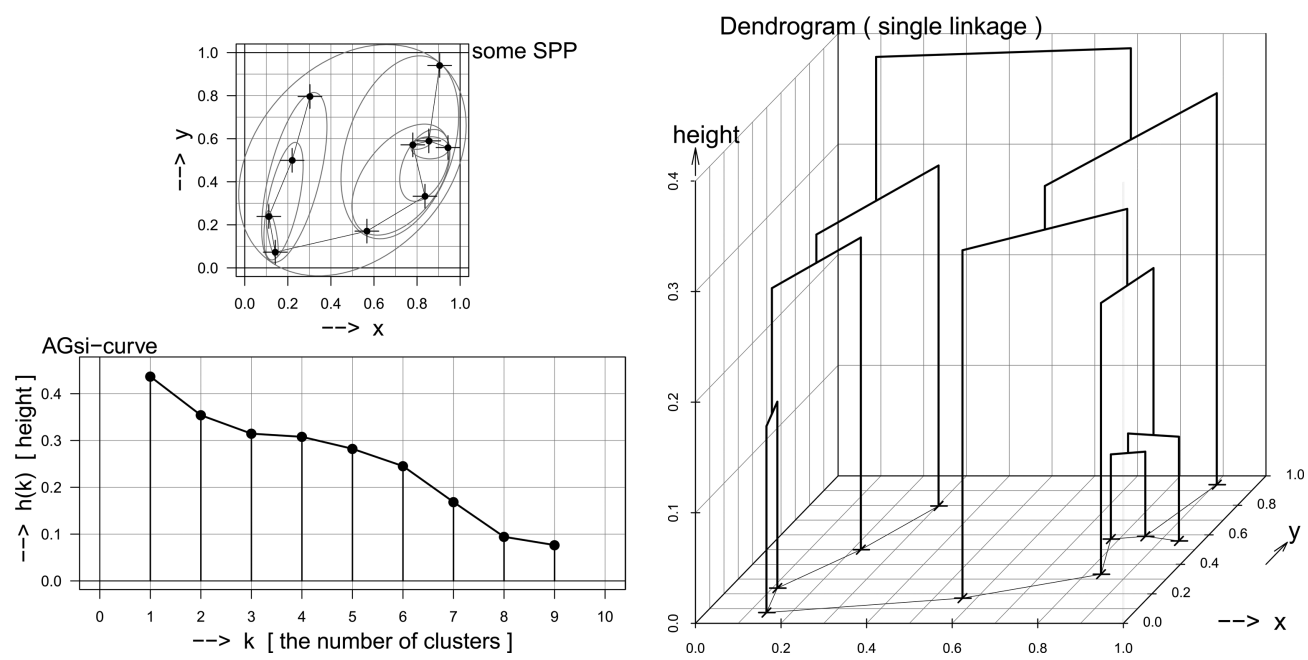

Fig. 1: A simple example of an AGsi-curve.

The proposed five-step procedure uses the AGsi-curve to classify SPPs with $n$ points in the unit cube in $R^{\nu}$.

[Step 1] Compute $m$ AGsi-curves for the SPP with $n$ points under CSR using $m$ sets of random numbers.

[Step 2] Plot the envelope corresponding to the $m$ AGsi-curves obtained in Step 1.

[Step 3] Plot the AGsi-curve for an observed SPP on the envelope obtained in Step 2.

[Step 4] Compare the curve in Step 3 to the envelope in Step 2.

[Step 5] Classify an observed SPP according to the result in Step 4.

If the curve is completely contained within the envelope, the SPP is classified as a random pattern.

If the curve departs from the envelope and gets close to a horizontal line, the SPP is classified as a regular pattern.

If the curve departs from the envelope and gets close to an L-shaped line, the SPP is classified as an aggregated pattern.

\subsubsection{Examples}

Three different types of SPPs with known distributions in a unit square $(\nu=2)$ are provided to illustrate the classification method.

The SPP names and properties are as follows:

Japanese black pine saplings $(n=65)$, a random pattern;

Biological cells $(n=42)$, a regular pattern;

Redwood seedlings $(n=62)$, an aggregated pattern.

These are shown in Figure 2 and were all introduced in Diggle (2003).

Diggle (2003) used the $G$-function to show that the SSPs Japanese black pine saplings, Biological cells, and Redwood seedlings are a random pattern, a regular pattern, and an aggregated pattern, respectively. Ripley (1977) also used the $K$-function to show that 

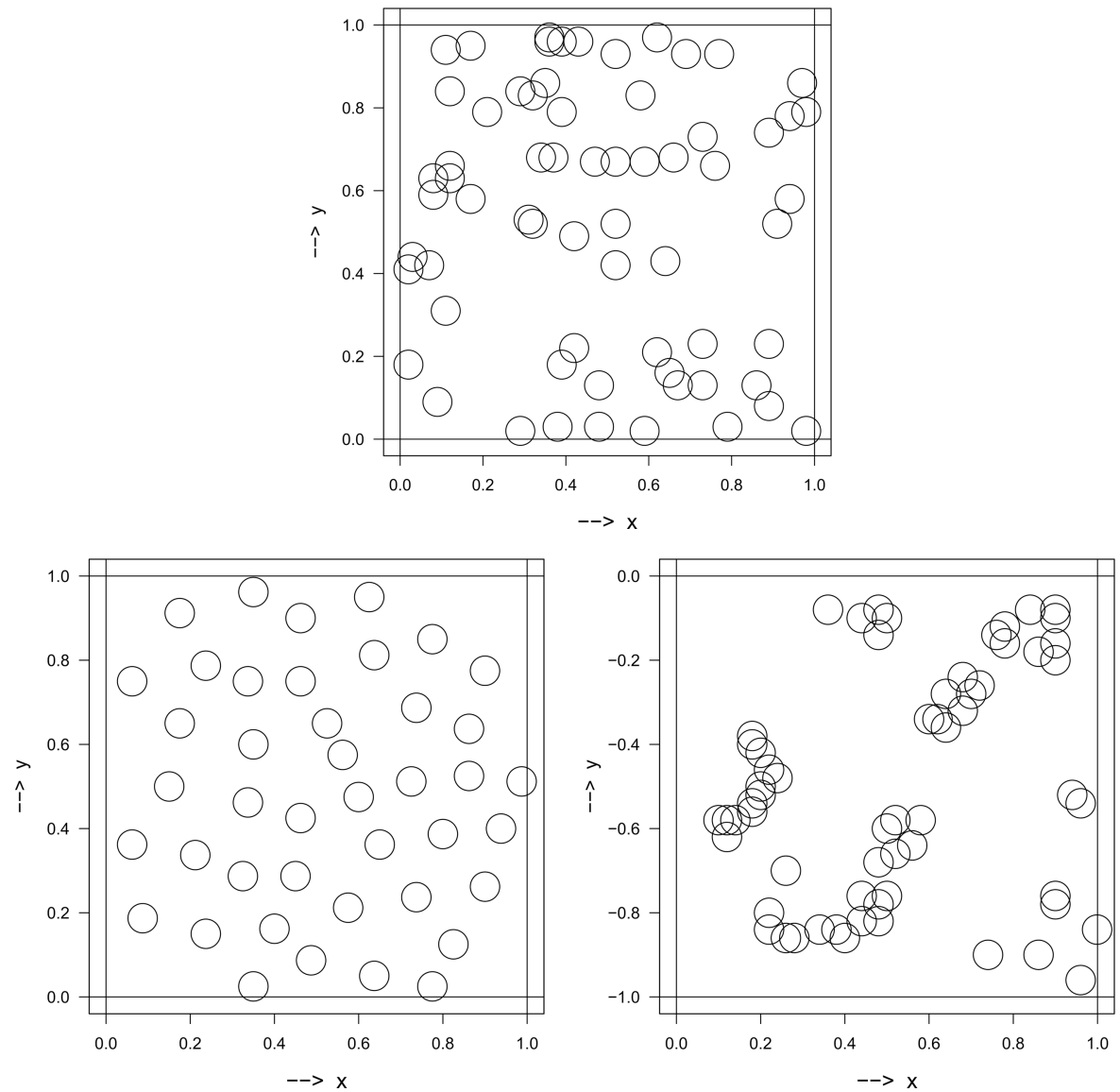

Fig. 2: Three real SPPs: Japanese black pine saplings (upper), Biological cells (lower left) and Redwood seedlings (lower right).

the Biological cells and Redwood seedlings SPPs are a regular pattern and an aggregated pattern, respectively.

The above three SPPs were classified by using the proposed procedure with $m=100$, and the results are shown in Figure 3. Their classification results matched those of Diggle (2003) and Ripley (1977). The Mersenne Twister pseudo random-numbers are used consistently in this simulation.

\section{Empirical comparisons of graphical methods}

In this section, we use artificially created SPPs to compare the AGsi-curve with the $G$ function and the $K$-function in order to clarify the different properties of these methods. Our results (presented below) show the advantage of the AGsi-curve over both the $G$-function and the $K$-function. 

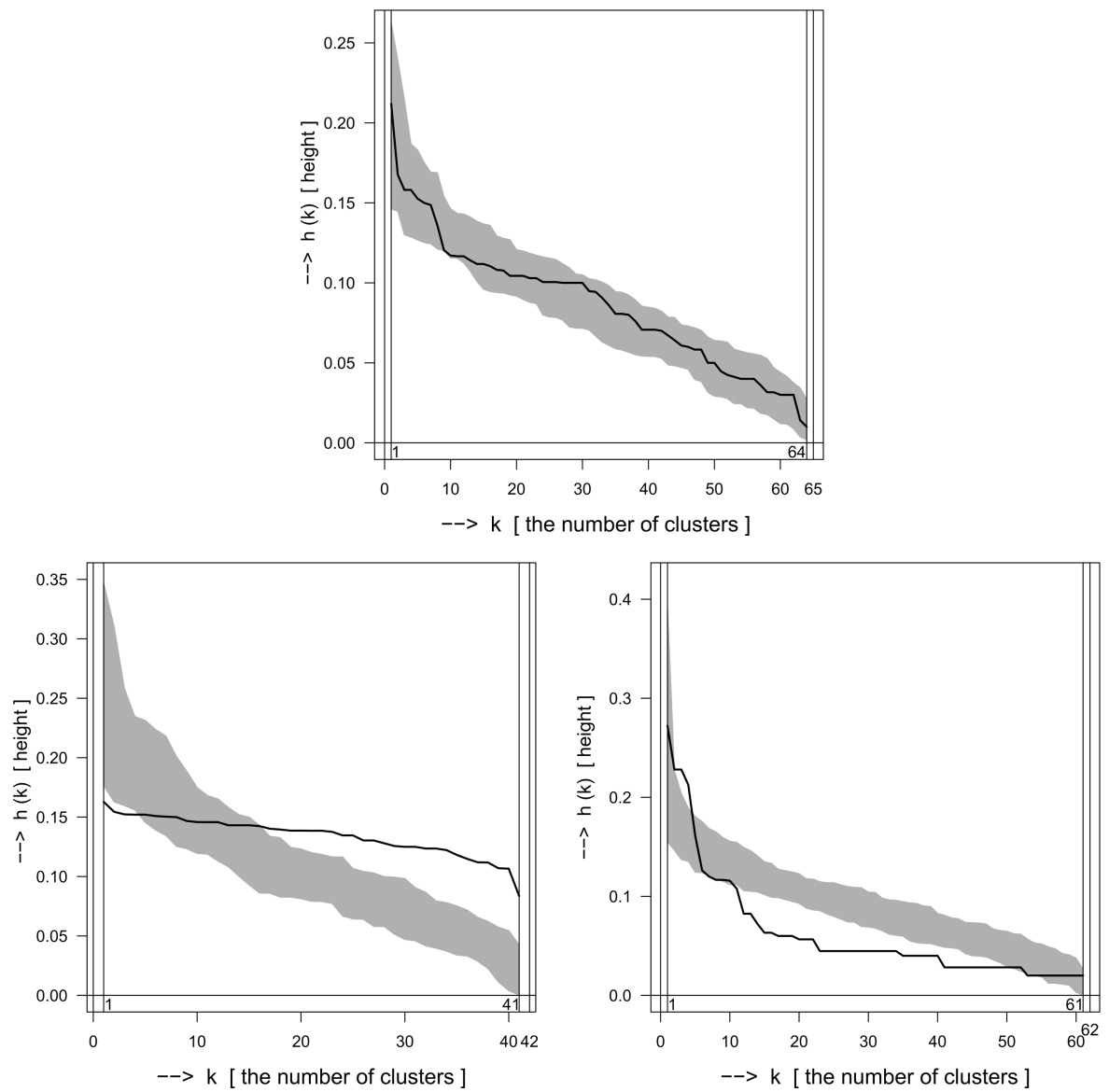

Fig. 3: Classification results using the AGsi-curve: Japanese black pine saplings (upper), Biological cells (lower left), Redwood seedlings (lower right).

\subsection{Comparison of the AGsi-curve and the $G$-function}

For this comparison, artificial SPPs were designed with $l \times l$ small square blocks $B_{1}, B_{2}, \ldots, B_{l \times l}$. The numbers of points in the corresponding blocks, $N_{1}, N_{2}, \ldots, N_{l \times l}$, are not necessarily the same. Here, in order to measure the distances in all blocks, we assumed $N_{1}, N_{2}, \ldots, N_{l \times l} \geq 2$. A square region was assembled from the small square blocks to produce various artificial SPPs, and we note that different types of SPPs can be represented by changing the configuration of the $l \times l$ small square blocks in the square region.

We define two measures for the distance between a block and the adjacent blocks. The first such measure is the point-to-point nearest-neighbor distance between blocks (Dbb). For a given block $B$, we define $\operatorname{Dbb}(B)$ as follows:

$$
\operatorname{Dbb}(B)=\min _{\substack{p \in B \\ q \in B^{a d j}}}\|p-q\|,
$$

where $p$ is a point belonging to $B, q$ is a point belonging to $B^{a d j}$, which is the set of all blocks adjacent to $B$, and \|\| is the Euclidean distance. The second measure is the point-to-point 
nearest-neighbor distance within the block (Dwb), which is defined as follows:

$$
\operatorname{Dwb}(B)=\min _{\substack{p, q \in B \\ p \neq q}}\|p-q\|
$$

where $p, q$ are different points in $B$. Dbb denotes the shortest distance between two points, where the points are in different blocks, Dwb denotes the distance from the $i$ th point to the nearest other point in the same block.

If $\operatorname{Dbb}(B)>\operatorname{Dwb}(B)$ regardless of the number of $l$, the linkage of nearest-neighbor pairs should be generated between points in the same block rather than points in different adjacent blocks. No matter what configuration is used for the $l \times l$ blocks in the entire square region, if $\operatorname{Dbb}(B)>\operatorname{Dwb}(B)$ holds for all $l \times l$ blocks, the point-to-point nearest-neighbor distances in the entire region are invariant. In this paper, 3 types of adjacent patterns are considered;

" placed at the corner ( surrounded by the other 3 blocks )",

"placed at the middle of the edge ( surrounded by the other 5 blocks )",

"placed at the center of the square ( surrounded by the other 8 blocks )".

A simple specific case of this for $l=4$ is shown below.

Two artificial SPPs are provided in Figure 4 for comparison of the AGsi-curve and the $G$-function. Both panels show that each entire region consists of $4 \times 4$ smaller blocks in a unit square, as follows: 12 blocks $\left(B_{1}, B_{2}, \ldots, B_{12}\right)$ include 2 points each, and another 4 blocks $\left(B_{13}, B_{14}, B_{15}, B_{16}\right)$ include 19 points each. By changing the configuration of the $4 \times 4$ smaller blocks, many different SPPs are produced. Figure 4 (left and right) shows two of them.
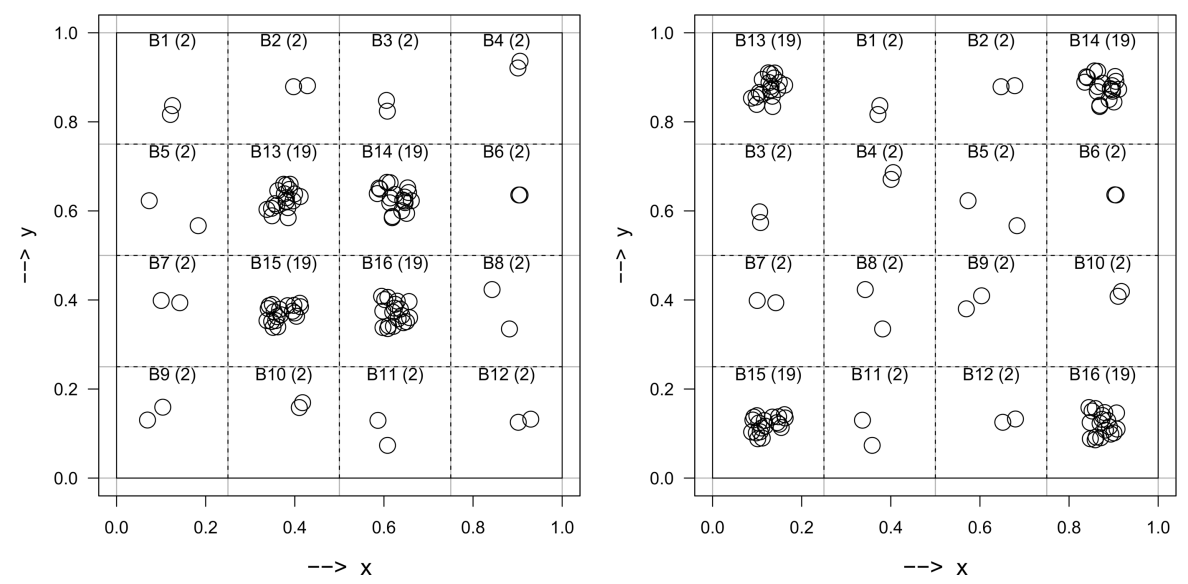

Fig. 4: Two different SPPs with $4 \times 4$ blocks: SPP1 (left), SPP2 (right).

Figure 5 (right) shows the results of applying the $G$-function to two different SPPs, SPP1 and SPP2. The $G$-function for SPP1 is exactly the same as the one for SPP2 because it is the $\mathrm{CDF}$ of the point-to-point nearest-neighbor distance for the entire region. Thus, the $G$-function cannot discriminate between SPP1 and SPP2. The simulation study of the $G$-function was conducted using the R package spatstat (version: 1.46-1) 

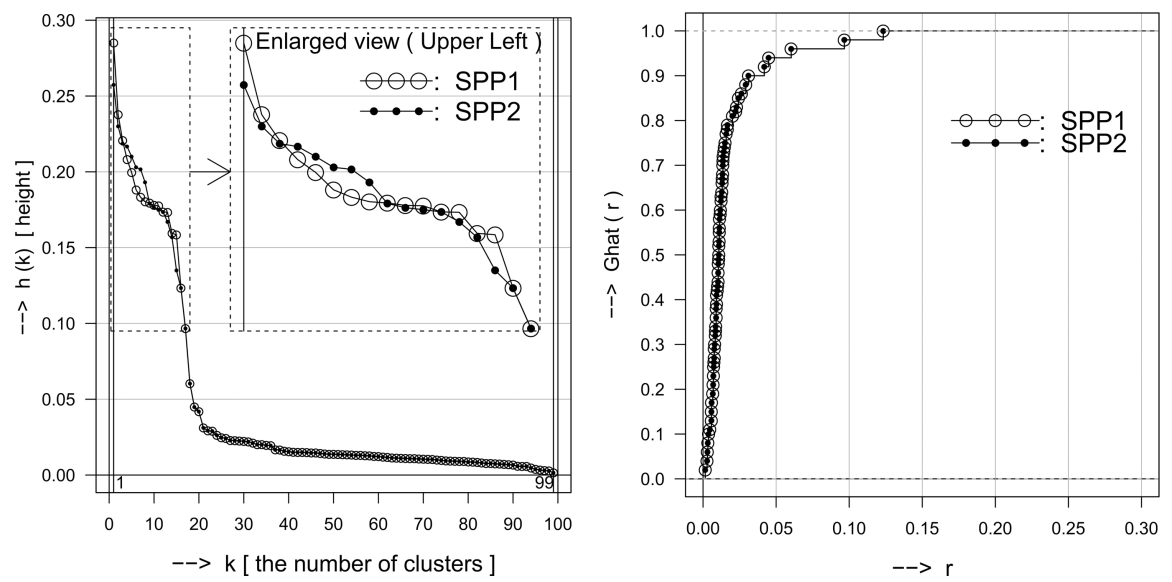

Fig. 5: The results of the AGsi-curve (left) and $G$-function (right) for SPP1 and SPP2.

On the other hand, the AGsi-curve necessarily links all points in the entire region and does not compare Dbb and Dwb, because it is based on a hierarchical cluster analysis. Consequently, the AGsi-curve does depend on the configuration of the $4 \times 4$ blocks. Figure 5 (left) shows the results of applying the AGsi-curve to the same two SPPs, SPP1 and SPP2. In a small number of clusters, the AGsi-curve for SPP1 is not the same as the one for SPP2, and thus, unlike the $G$-function, the AGsi-curve is able to discriminate between SPP1 and SPP2.

\subsection{Comparison of the AGsi-curve and the $K$-function}

To compare the AGsi-curve and the $K$-function, we used an artificial regular SPP with 200 points in a unit square; it was formed by a simple sequential inhibition (SSI) process (Diggle, 1976) with a radius of inhibition equal to 0.0375 . The artificial regular SPP shown in Figure 6 was classified using the AGsi-curve by following the procedure presented in

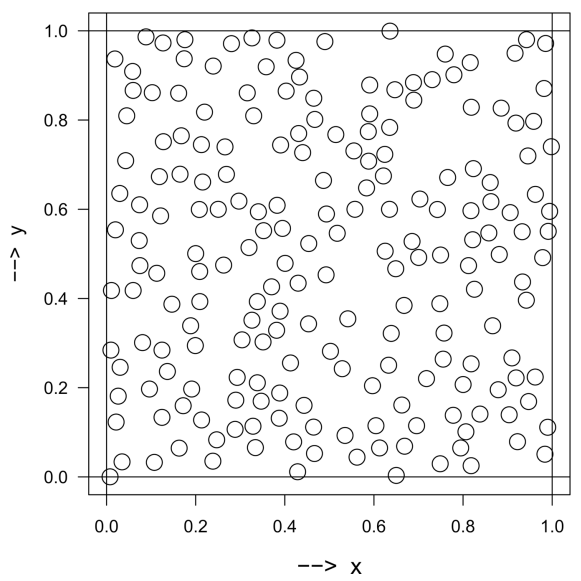

Fig. 6: A realization of an SSI with 200 points and with a radius of inhibition equal to 0.0375 . 
Section 2.2 .

Figure 7 shows the results of applying the AGsi-curve and the $K$-function to the simulated SSI process with upper and lower simulation envelopes and with $m=100$ simulations of a CSR process. The AGsi-curve (left) for the SSI process exceeds most of the CSR envelopes, while the $K$-function (right) estimate for the SSI process is almost contained in the CSR envelope, except for the small $r$ region. Therefore, the AGsi-curve shows clearer deviation from the CSR envelope than the $K$-function in this case. The simulation study of the $K$-function was conducted using the R package spatstat (version: 1.46-1).
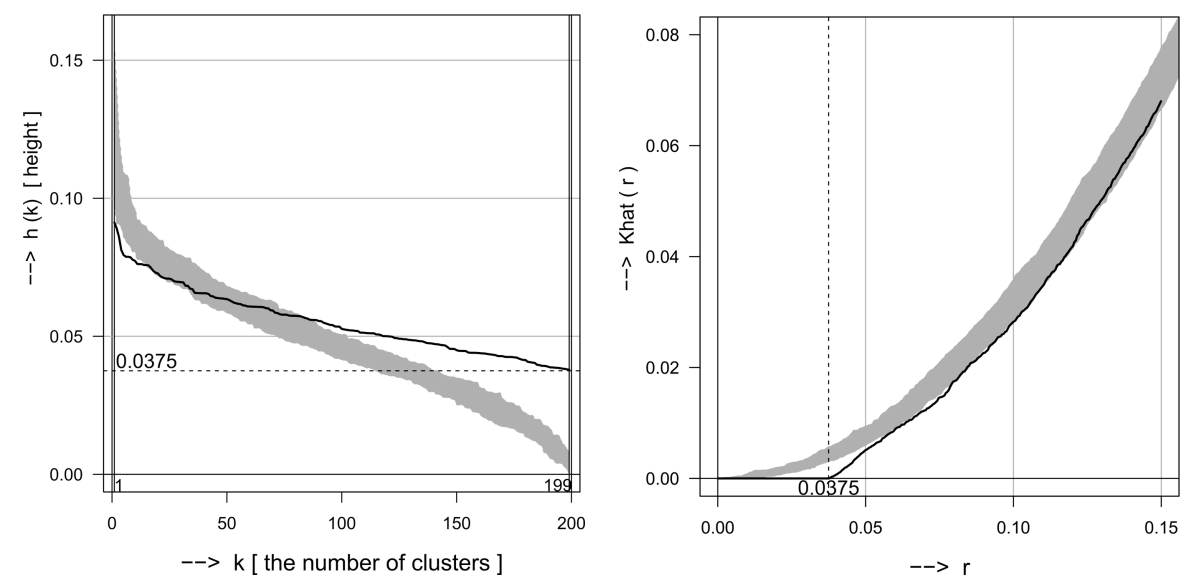

Fig. 7: The results of the AGsi-curve (left) and $K$-function (right).

\subsection{Robustness against the edge effects}

In spatial point analysis, there has been pointed out the edge effects problem which occurs when the search area intercepts the edge of the study region (Cressie, 1991). As will be shown below, the AGsi-curve and the $G$-function are more robust against the edge effects than the $K$-function. To confirm this property, the AGsi-curve, $G$-function and the $K$-function without the edge correction are compared in terms of the edge effects. For this comparison, two artificial SPPs with different shaped regions are provided in Figure 8 (left and right). One of them is the square-shaped region with area 1 , and the other is the rectangle-shaped region with area 1 . Both of them have 100 points under CSR in the region. 100 sets of SPPs are generated in each region using random numbers, and the simulation envelope for them is computed.

The colorless envelope corresponding to the rectangle-shaped region is displayed superimposed on the gray envelope corresponding to the square-shaped region for the AGsi-curve, the $G$-function and the $K$-function, respectively. The resulting diagrams are provided in Figure 9. The upper panel shows the $K$-function without the edge correction. The lower left panel shows the AGsi-curve, and the lower right panel shows the $G$-function. The mean levels for two groups of AGsi-curves almost coincide as well as those of $G$-functions, in contrast with the apparent difference in those of $K$-functions. For reference, the envelopes of $K$-functions with Ripley's edge correction are provided in Figure 10. 
TAKAI, TAMURA and MOTOYAMA
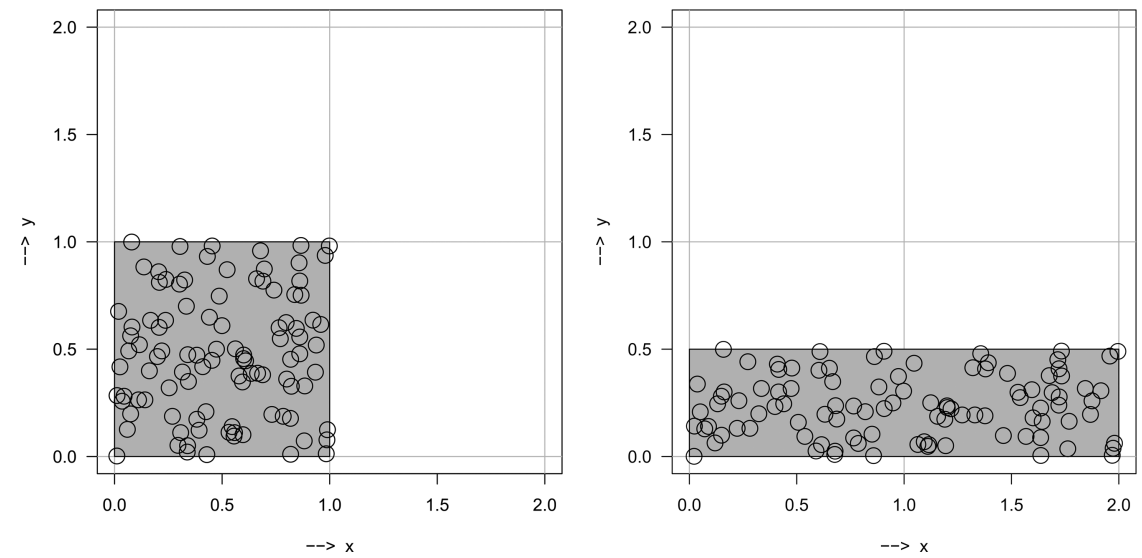

Fig. 8: Two artificial SPPs: the square-shaped regin (left) and the rectangle-shaped region (right). Both of them have 100 points under CSR.
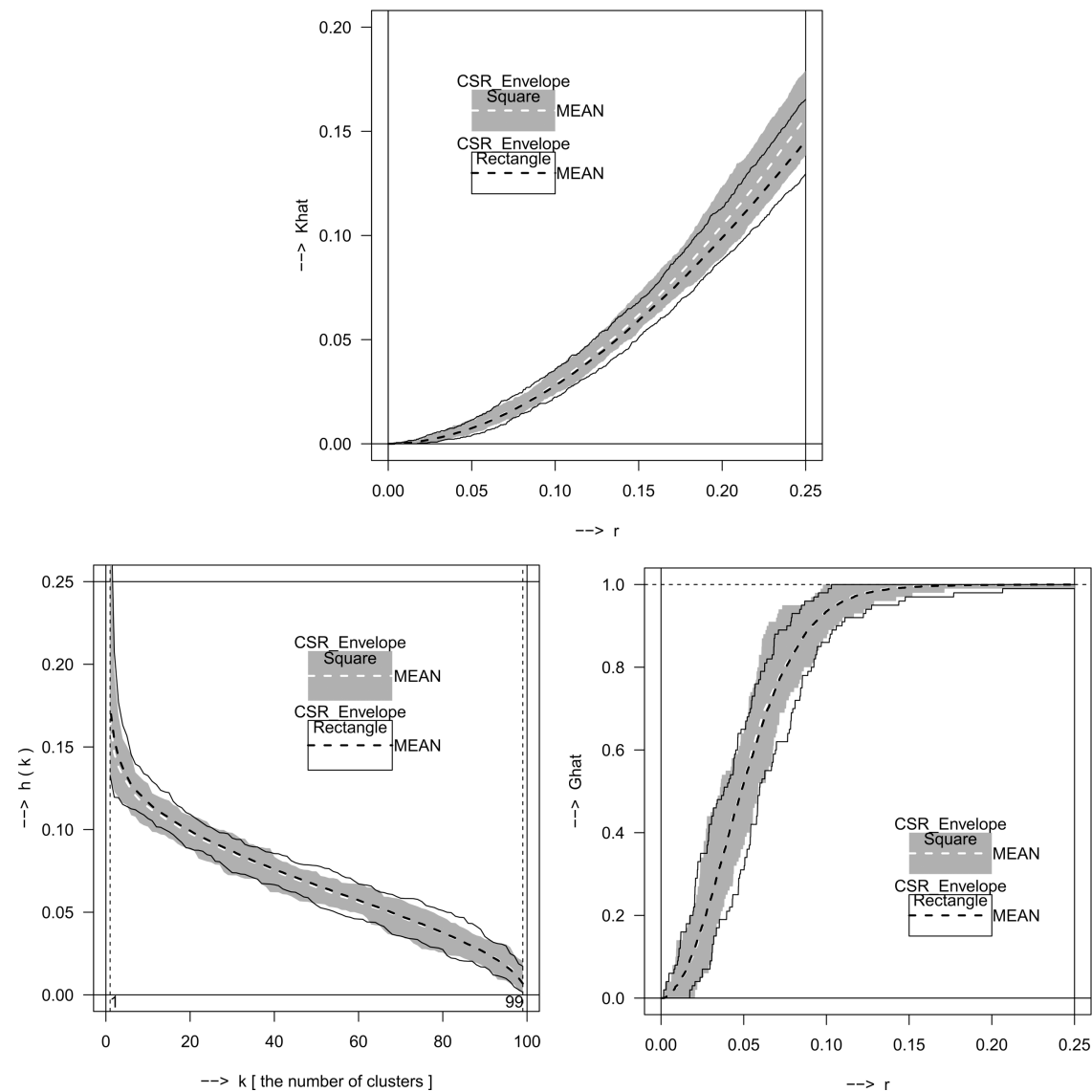

Fig. 9: The $K$-function without the edge correction (upper), the AGsi-curve (lower left) and the $G$-function (lower right) for two artificial APPs (Fig. 8). 


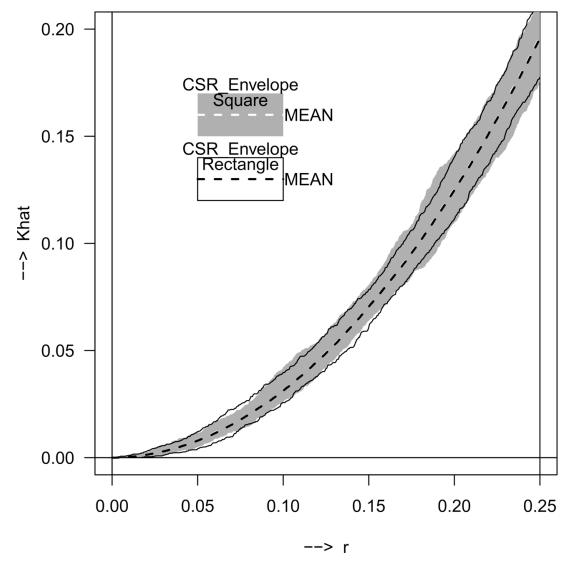

Fig. 10: The $K$-function with Ripley's edge correction for two artificial APPs (Fig. 8).

\section{Discussion}

This section discusses the relationship between the AGsi-curve and the MST. Let $D(1)<D(2)<\cdots<D(k)<\cdots<D(n-2)<D(n-1)$ denote the ordered lengths of the graph edges of the MST (GEMST) for $n$ points in some region. From the definition of the AGsi-curve, the sizes of $h(k)$ have the following order: $h(n-1)<h(n-2)<\cdots<$ $h(k)<\cdots<h(2)<h(1)$. According to Gower and Ross (1969), $h(k)=D(n-k)$ holds for all $k=1,2, \ldots, n-1$. Therefore, the lengths of the GEMST and $h(k)$ have a monotonic oneto-one correspondence, but in the reverse order. We will show that the AGsi-curve contains information that is equivalent to that of the empirical CDF of the lengths of the GEMST.

Let $\{(1, h(1)),(2, h(2)), \ldots,(k, h(k)), \ldots,(n-2, h(n-2)),(n-1, h(n-1))\}$ denote the coordinates of both the ends and the kinked points of the AGsi-curve. $F_{n}(x)$ can be constructed using the information of these coordinates, as follows:

$$
F_{n}(x)=\left\{\begin{aligned}
0, & 0 \leq x<h(n-1) \\
\frac{1}{n-1}, & h(n-1) \leq x<h(n-2) \\
\frac{2}{n-1}, & h(n-2) \leq x<h(n-3) \\
\cdots & \cdots \\
\frac{n-k}{n-1}, & h(n-k) \leq x<h(n-k-1) \\
\cdots & \cdots \\
\frac{n-2}{n-1}, & h(2) \leq x<h(1) \\
\frac{n-1}{n-1}=1, & h(1) \leq x \\
0 & \leq x<\infty .
\end{aligned}\right.
$$

Clearly, $F_{n}(x)$ is the empirical CDF of $h(1), h(2), \ldots, h(k), \ldots, h(n-2), h(n-1)$.

Conversely, given $F_{n}(x)$, its inverse $F_{n}^{-1}(u)$ is defined as follows:

$$
F_{n}^{-1}(u)=\inf \left\{x: F_{n}(x) \geq u\right\} \quad 0 \leq u \leq 1
$$

and $h(1), h(2), \ldots, h(k), \ldots, h(n-2), h(n-1)$ can be calculated as follows: 
TAKAI, TAMURA and MOTOYAMA

$$
\left\{\begin{array}{r}
h(1)=F_{n}^{-1}(1) \\
h(2)=F_{n}^{-1}\left(\frac{n-2}{n-1}\right) \\
\cdots \\
h(k)=F_{n}^{-1}\left(\frac{n-k}{n-1}\right) \\
\cdots \\
h(n-2)=F_{n}^{-1}\left(\frac{2}{n-1}\right) \\
h(n-1)=F_{n}^{-1}\left(\frac{1}{n-1}\right) .
\end{array}\right.
$$

A sequence of points $\{(1, h(1)),(2, h(2)), \ldots,(k, h(k)), \ldots,(n-2, h(n-2)),(n-1, h(n-1))\}$ is obtained. The AGsi-curve can be constructed by connecting these points in order.

A simple example of this is shown in Figure 11. The upper panel shows the MST and
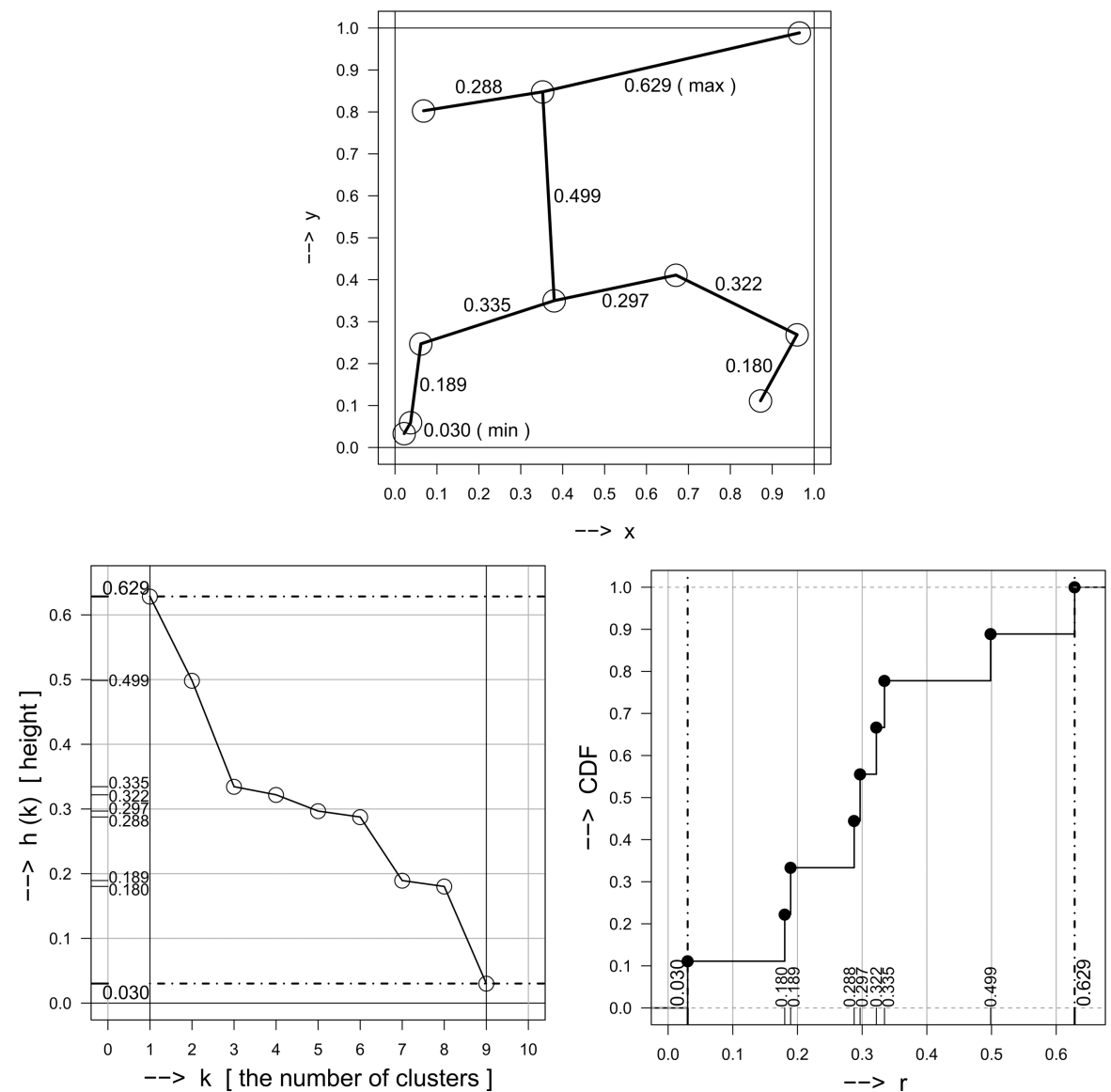

Fig. 11: The AGsi-curve (lower left) and the empirical CDF of the lengths of the GEMST (lower right) for the given SPP (upper). 
the length of each GEMST for an SPP that has ten points in the unit square. The lower-left panel shows the AGsi-curve for the SPP, and the lower-right panel shows the empirical CDF of the lengths of the GEMST of the SPP. The simulation of the MST was conducted using the $\mathrm{R}$ package vegan (version: 2.4-1).

\section{Conclusion}

This paper proposed a new graphical method to classify SPPs. This method is called the AG-curve, which is obtained by converting the information contained in the dendrogram of a hierarchical cluster analysis.

The research was confined almost exclusively to the AGsi-curve which is a variant of the AG-curve; a procedure for obtaining the AGsi-curve and how to use it to classify an SPP were discussed and demonstrated. And also it was showed that the classification performance of the AGsi-curve is as good or better than that of other graphical methods.

$h(k)$ s can be calculated not only by the single-linkage method like this paper but also by the other hierarchical clustering methods. If the mathematical properties of other $h(k) \mathrm{s}$ will be revealed, some of the ideas in this paper can be extended to other types of AG-curves, such as the AGco-curve and the AGav-curve.

\section{Acknowledgement}

The authors would like to thank the editor, an associate editor and two anonymous referees for their helpful suggestions that improved the quality of the original manuscript.

\section{REFERENCES}

Cressie, N. A. C. (1991). Statistics for Spatial Data, John Wiley \& Sons, Inc., New York.

Diggle, P. J., Besag, J. \& Gleaves, J.T. (1976). Statistical analysis of spatial point patterns by means of distance methods. Biometrics, 32, 659-667.

Diggle, P. J. (1979). On parameter estimation and goodness-of-fit testing for spatial point patterns. Biometrics, 35, 87-101.

Diggle, P.J. (2003). Statistical Analysis of Spatial Point Pattern, 2nd edition. Academic Press, New York.

Diggle, P.J. (2013). Statistical Analysis of Spatial and Spatio-Temporal Point Patterns, 3rd edition. Chapman and Hall/CRC.

Dixon, P. M. (2002). Ripley's $K$ function, in. Encyclopedia of Environmetrics, Vol.3, Ed. by. A.H. El-Shaarawi and W. W. Piegorsch. John Wiley \& Sons, Ltd, Chichester, pp.17961803.

Duranton, G., \& Overman, H. G. (2005). Testing for localization using micro-geographic data. The Review of Economic Studies, 72(4), 1077-1106.

Gatrell, A. C., Bailey, T. C., Diggle. P. J., \& Rowlingson, B. S. (1996). Spatial point pattern analysis and its application in geographical epidemiology, Trans Inst Br Geogr NS, 21, 256-274.

Gower, J. C., Ross, J. S. (1969). Minimum Spanning Trees and Single Linkage Cluster Analysis. Journal of the Royal Statistical Society, Series C. 18(1), 54-64.

Johnson, S. C. (1967). Hierarchical clustering schemes. Psychometrika, 32, 241-254.

Kruskal, J. B. (1956). On the shortest spanning subtree of a graph and a traveling salesman problem. Proc. Amer. Math. Soc., 7, 48-50. 
Lance, G.N., \& Williams, W. T. (1966). Computer programs for hierarchical polythetic classification ("similarity analyses"). The Computer Journal, 9(1), 60-64.

Lance, G. N., \& Williams, W. T. (1967). A general theory of classificatory sorting strategies: 1. Hierarchical systems. The Computer Journal, 9(4), 373-380.

Ogata, Y. (1999). Seismicity analysis through point-process modeling: A review, Pure Appl. Geophys, 155, 471-507.

Okabe, A., \& Yamada, I. (2001). The $K$-function method on a network and its computational implementation. Geographical Analysis, 33(3), 271-290.

Pereira (2003) Analysis of spatial point patterns using hierarchical clustering algorithms (http://research-repository.uwa.edu.au/files/3240537/Pereira_Sandra_M._C._2003.pdf)

Perry, G. L. W., Miller, B. P., \& Enright, N. J. (2006). A comparison of methods for the statistical analysis of spatial point patterns in plant ecology. Plant Ecology 187: 59-82.

Ripley, B.D. (1976). The Second-Order Analysis of Stationary Point Processes. Journal of Applied Probability, 13, 255-266.

Ripley, B.D. (1977). Modelling spatial patterns (with Discussion). Journal of the Royal Statistical Society, Series B, 39, 172-212.

Ripley, B.D. (1979). Tests of 'randomness' for spatial point patterns, Journal of the Royal Statistical Society, Series B. 41, 368-374.

Waagepetersen, R., Guan, Y., Jalilian, A., Mateu, J. (2016). Analysis of multispecies point patterns by using multivariate log-Gaussian Cox processes. Journal of the Royal Statistical Society, Series C. 65, 77-96.

Zimmerman, D.L. (1993). A Bivariate Cramer-Von Mises Type of Test for Spatial Randomness. Applied Statistics, 42, 43-54.

(Received: November 22, 2016, Accepted: May 9, 2017) 